\title{
Enantiomer-Selective Radical Polymerization of rac-2,4-Pentanediyl Dimethacrylate by 2,2'-Azobisisobutyronitrile/Copper(II) Trifluoromethanesulfonate/Chiral Diamine as Asymmetric Reverse Atom Transfer Radical Polymerization Initiating System
}

\author{
Masashi Tsuji, Ryosuke SAKAI, Toshifumi SATOH, Harumi KAGA, ${ }^{*}$ and Toyoji KAKUCHI ${ }^{\dagger}$ \\ Division of Molecular Chemistry, Graduate School of Engineering, Hokkaido University, Sapporo 060-8628, Japan \\ *National Institute of Advanced Industrial Science and Technology (AIST), 2-17-2-1 Tsukisamu-Higashi, \\ Toyohira, Sapporo 062-8517, Japan
}

(Received September 6, 2002; Accepted November 15, 2002)

KEY WORDS Atom Transfer Radical Polymerization (ATRP) / Enantiomer-Selective Polymerization / Cyclopolymerization /

Asymmetric polymerization, such as asymmetric synthesis polymerization, helix-sense selective polymerization, and enantiomer-selective polymerization, is of significant interest from the viewpoint of the precise control of a polymerization system. In general, ionic and coordination polymerization systems were easily modified into chiral ones, so that they were used for preparing various types of asymmetric polymerizations, e.g., high enantiomer-selectivity was achieved for the anionic polymerization of the racemic $\alpha$-monosubustitued benzyl methacrylate using the (-)-sparteine/Grignard reagent ${ }^{1-3}$ and the ringopening polymerization of propylene sulfide with (-)binaphthol/ZnEt ${ }_{2}{ }^{4,5}$ Although the radical polymerization is also available for asymmetric polymerization, little is known about the enantiomer-selective radical polymerization. ${ }^{6,7}$

Recently, we reported the enantiomer-selective radical cyclopolymerization of rac-2,4-pentanediyl dimethacrylate (rac-1) using the atom transfer radical polymerization (ATRP) initiating system modified with chiral ligands, i.e., the methyl 2bromoisobutyrate $/ \mathrm{CuBr} / \mathrm{chiral}$ amine ligand. ${ }^{8}$ Matyjaszewski has expanded the ATRP initiating system into the "reverse" ATRP initiating system such as the 2,2'-azobisisobutyronitrile (AIBN)/copper(II) trifluoromethanesulfonate $\left(\mathrm{Cu}(\mathrm{OTf})_{2}\right) /$ amine ligand. ${ }^{9}$ Therefore the significant interest is to examine the "reverse" ATRP initiating system as a chiral one for the asymmetric polymerization. Here, we report the enantiomer-selective radical cyclopolymerization of $r a c-1$ using the $\mathrm{AIBN} / \mathrm{Cu}(\mathrm{OTf})_{2} /$ chiral diamine ligand such as $6,6^{\prime}$-bis $\{(1 R, 4 R)$-hydroxy-1,7,7-trimethylbicyclo[2.2.1] heptan-2-endo-yl\}-2,2'-bipyridine (3), (-)-sparteine (4), 3-bis[4' - $(S)$-isopropyloxazoline$2^{\prime}$-yl]pyridine $(S-5)$, and 3-bis[4'-(R)-isopropyloxazoline- $2^{\prime}$-yl]pyridine $(R-\mathbf{5})$ as the asymmetric reverse ATRP initiating system, as shown in Scheme 1.

\section{RESULTS AND DISCUSSION}

Table I lists the results for the polymerization of rac-1 using the initiating system consisting of AIBN, $\mathrm{Cu}(\mathrm{OTf})_{2}$, and 3, 4, $S-\mathbf{5}$, and $R-\mathbf{5}$ as chiral ligands. All polymerizations homogeneously proceeded to yield gel-free polymers. The characteristic resonance due to the methacrylic groups was not observed in the ${ }^{1} \mathrm{H}$ and ${ }^{13} \mathrm{C}$ NMR spectra of the obtained polymers, indicating that the polymerization of $\mathrm{rac}-\mathbf{1}$ proceeded through a cyclopolymerization mechanism to afford the polymer essentially consisting of the cyclic repeating units, i.e., the extent of cyclization was $c a$. $100 \%$. The number average molecular weights $\left(M_{\mathrm{n}} \mathrm{s}\right)$ of the resulting polymers ranged from 47900 to 59100 and the polydispersity indices $\left(M_{\mathrm{w}} / M_{\mathrm{n}} \mathrm{s}\right)$ were $1.41-2.53$.

When 3 and $S$-5 were used as the chiral ligands, $(2 S, 4 S)$-2,4-pentanediyl dimethacrylate (SS-1) was predominantly polymerized, resulting in the fact that the enantiomeric excess (e.e.) of the recovered monomer for 3 was $4.2 \%$ for a monomer conversion of $22.8 \%$ and that for $S-5$, it was $2.3 \%$ for a monomer conversion of $18.3 \%$. It should be noted that $(2 R, 4 R)$ 2,4-pentanediyl dimethacrylate $(R R-1)$ was predominantly polymerized using 2-bromoisobutyrate/ $\mathrm{CuBr} / \mathbf{3}$

${ }^{\dagger}$ To whom correspondence should be addressed (Tel and Fax: +81-11-706-6602, E-mail: kakuchi@poly-mc.eng.hokudai.ac.jp). 

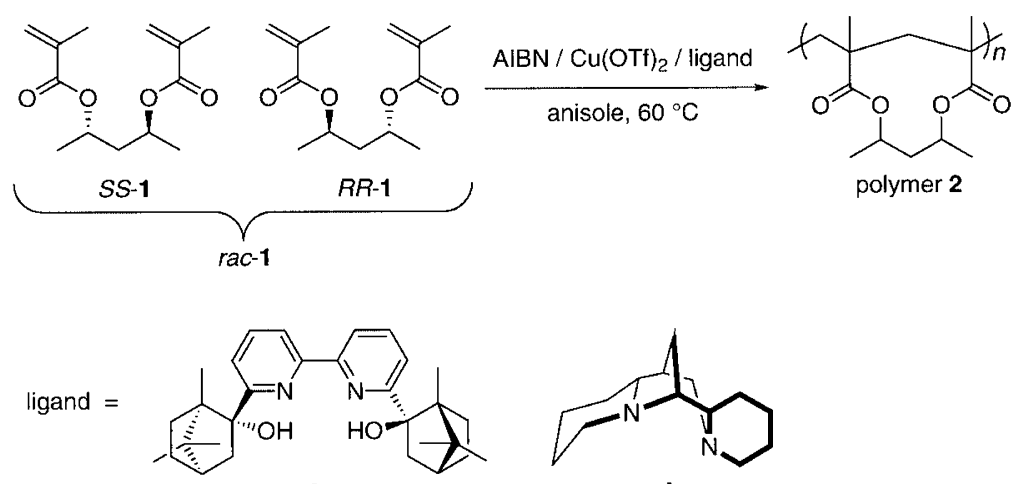

3

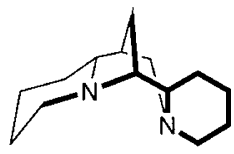

4<smiles>CC(C)(C)C1COC(c2cccc(C3=NC(C(C)(C)C)CO3)n2)=N1</smiles><smiles>CC(C)(C)C1COC(c2cccc(C3=NC(C(C)(C)C)CO3)n2)=N1</smiles>

S-5 $R-5$

Scheme 1 .

Table I. Polymerization of $r a c-1$ using AIBN $/ \mathrm{Cu}(\mathrm{OTf})_{2} /$ ligand initiation system $^{\mathrm{a}}$

\begin{tabular}{|c|c|c|c|c|c|c|c|}
\hline \multirow{2}{*}{ ligand } & \multirow{2}{*}{$\begin{array}{l}\text { time } \\
\mathrm{hr}\end{array}$} & \multirow{2}{*}{$\begin{array}{c}\text { conv. }^{b} \\
\%\end{array}$} & \multicolumn{2}{|c|}{$\begin{array}{l}\text { recovered } \\
\text { monomer }\end{array}$} & \multicolumn{2}{|c|}{ polymer $\mathbf{2}$} & \multirow{2}{*}{$r^{\mathrm{f}}$} \\
\hline & & & $\begin{array}{l}\text { selected } \\
\text { monomer }\end{array}$ & $\begin{array}{c}e^{e . e . .^{\mathrm{b}, \mathrm{c}}} \\
\%\end{array}$ & $M_{\mathrm{n}}\left(M_{\mathrm{w}} / M_{\mathrm{n}}\right)^{\mathrm{d}}$ & $\begin{array}{c}{[\alpha]_{435^{\mathrm{e}}}} \\
\text { deg. }\end{array}$ & \\
\hline 3 & 0.25 & 22.8 & $S S-1$ & 4.2 & $47900(2.53)$ & +13.6 & 2.0 \\
\hline 4 & 12 & 18.8 & $R R-\mathbf{1}$ & 9.4 & $56300(1.41)$ & -12.6 & 1.7 \\
\hline$S-5$ & 3 & 18.3 & $S S-1$ & 2.3 & $54500(1.55)$ & +11.3 & 1.2 \\
\hline$R-5$ & 2 & 18.5 & $R R-1$ & 4.6 & $59100(1.49)$ & -12.1 & 1.4 \\
\hline
\end{tabular}
vent, anisole; temperature $60{ }^{\circ} \mathrm{C}$. ${ }^{b}$ Determined by HPLC equipped with CHIRALCEL OB-H column. $\quad{ }^{\mathrm{c}}$ Enantiomeric excess of recovered monomer. ${ }^{\mathrm{d}}$ Determined by GPC in THF using polystyrene standards. ${ }^{\mathrm{e}}$ Measured in chloroform $(c 0.3$, $\left.28^{\circ} \mathrm{C}\right) .{ }^{\mathrm{f}}$ Enantiomer selectivity ratio $(r=\ln \{(1-$ conv. $/ 100)(1-$ e.e. $/ 100)\} / \ln \{(1-$ conv. $/ 100)(1+$ e.e. $/ 100)\})^{3}$.

or $S-\mathbf{5}$ as a normal ATRP initiating system, which was previously reported. ${ }^{8}$ The cause of the contradiction in enantiomer-selectivity is obscure, which should be clarified in our near study. On the other hand, $R R$ 1 was preferentially polymerized with $9.4 \%$ e.e. at a $18.8 \%$ monomer conversion and $4.6 \%$ e.e. at a $18.5 \%$ monomer conversion, using 4 and $R-5$, respectively. In addition, the enantiomer selectivity apparently changed with the chirality of the ligands used, e.g., the SS-1 enantiomer predominantly polymerized for $S-5$, while the $R R-1$ enantiomer polymerized for $R-\mathbf{5}$. The resulting polymers exhibited optical activity, and the specific rotations $\left([\alpha]_{435}, c 0.3\right.$, in chloroform) of the resulting polymers were $-12.6^{\circ} \sim+13.6^{\circ}$, corresponding to the optical activity of the predominantly polymerized enantiomer. These results indicated that the asymmetric reverse ATRP initiating system was effective for the enantiomer-selective radical cyclopolymerization leading to the optically active polymer.

For the polymerization of rac-1 using the AIBN/

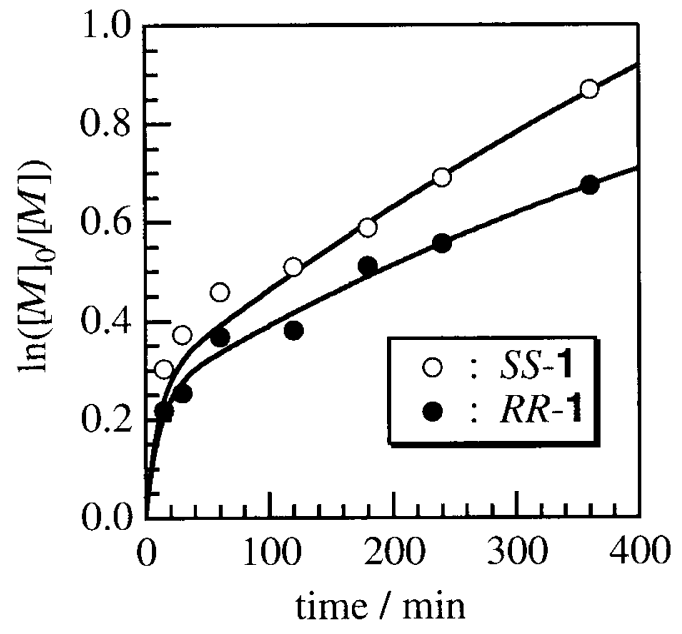

Figure 1. Kinetic plots for the polymerization of $\mathrm{rac}-\mathbf{1}$ using $\mathrm{AIBN} / \mathrm{Cu}(\mathrm{OTf})_{2} / \mathbf{3}$ initiating system.

$\mathrm{Cu}(\mathrm{OTf})_{2} / 3$ initiating system, the kinetic plots are shown in Figure 1. The reaction rate of the SS-1 enantiomer was larger than that of the $R R-\mathbf{1}$ enantiomer, and 


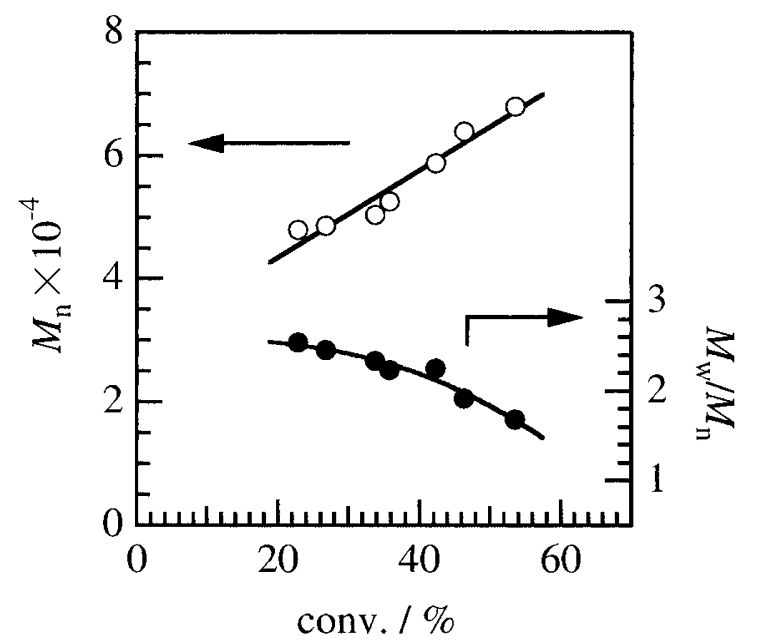

Figure 2. Dependence of $M_{\mathrm{n}}$ and $M_{\mathrm{w}} / M_{\mathrm{n}}$ on monomer conversion for the polymerization of $\mathrm{rac}-\mathbf{1}$ using $\mathrm{AIBN} / \mathrm{Cu}(\mathrm{OTf})_{2} / \mathbf{3}$ initiating system.

the enantiomer selectivity ratio $r$ was 2.0 at a $22.8 \%$ monomer conversion. Figure 2 shows the relationships between $M_{\mathrm{n}}$ and $M_{\mathrm{w}} / M_{\mathrm{n}} v s$. the monomer conversion. The $M_{\mathrm{n}}$ values of the resulting polymers linearly increased with the monomer conversion, though these values were greater than the calculated ones, e.g., for conversion $=22.8 \%$; the observed and calculated values were 47900 and 10900 , respectively. The $M_{\mathrm{w}} / M_{\mathrm{n}}$ values decreased with the increasing monomer conversion. Figure 3 shows the relationships between the e.e. value of the recovered monomer and the $[\alpha]_{435}$ value of the resulting polymer vs. the monomer conversion. The increasing monomer conversion tended to increase the e.e. value of the recovered monomer. On the other hand, the $[\alpha]_{435}$ value of the resulting polymer decreased with the increasing monomer conversion, because the optical purity (o.p.) of the polymer decreased with the increase in the monomer conversion, e.g., the o.p. value of the resulting polymer was $14.2 \%$ at a $22.8 \%$ monomer conversion and $2.1 \%$ at a $59.6 \%$ monomer conversion. ${ }^{3}$ These results indicated that the chiral copper complex should affect the addition of rac1 to the growing end, in which the SS-1 enantiomer was predominantly polymerized, i.e., the enantiomerselective radical polymerization based on the reverse ATRP.

In summary, we achieved the enantiomer-selective radical polymerization by the cyclopolymerization of rac-2,4-pentanediyl dimethacrylate ( $r a c-1)$ based on the reverse atom transfer radical polymerization (reverse ATRP) method using chiral initiating systems. For $\mathrm{AIBN} / \mathrm{Cu}(\mathrm{OTf})_{2} / 6,6^{\prime}$-bis $\{(1 R, 4 R)-$ hydroxy-1,7,7-trimethylbicyclo[2.2.1]heptan-2-endoyl $\}$-2,2'-bipyridine (3), the $(2 S, 4 S)$-2,4-pentanediyl dimethacrylate $(S S-1)$ enantiomer predominantly poly-

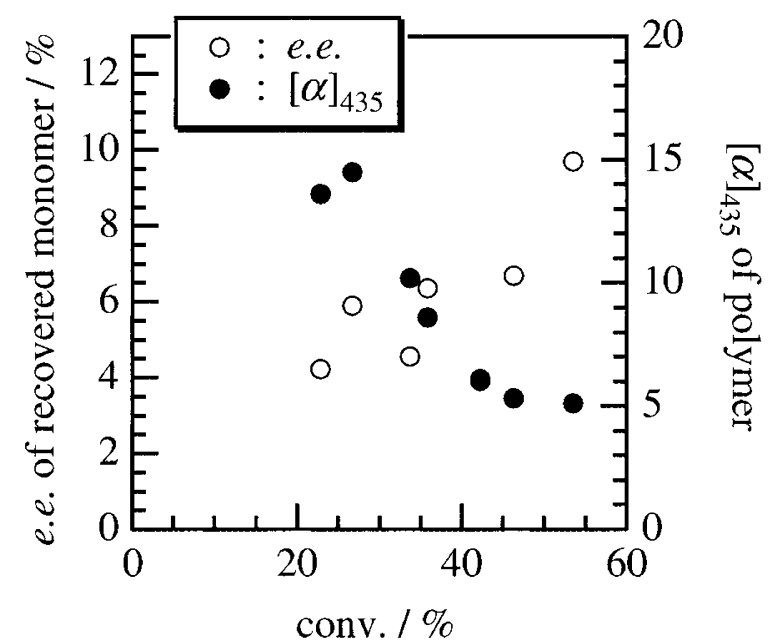

Figure 3. Dependence of e.e. of recovered monomer and $[\alpha]_{435}$ of resulting polymer on monomer conversion for the polymerization of $r a c-1$ using $\mathrm{AIBN} / \mathrm{Cu}(\mathrm{OTf})_{2} / 3$ initiating system.

merized, and the enantiomeric excess of the recovered monomer increased with the increasing monomer conversion.

\section{EXPERIMENTAL}

\section{Materials}

2,2'-Azobisisobutyronitrile (AIBN) was recrystallized from methanol. Dry-anisole, 3-bis[4'-(S)-isopropyloxazoline-2'-yl]pyridine, 3-bis[4'-(R)-isopropyloxazoline- $2^{\prime}$-yl]pyridine and trifluoromethanesulfonate $\left(\mathrm{Cu}(\mathrm{OTf})_{2}\right)$ were purchased from the Aldrich Chem. Co., and used without further purification. (-)-Sparteine was purchased from the Aldrich Chem. Co. and distilled over $\mathrm{CaH}_{2}$ under reduced pressure. $\quad 6,6^{\prime}$-Bis $\{(1 R, 4 R)$-hydroxy-1,7,7-trimethylbicyclo[2.2.1]-heptan-2-endo-yl\}-2,2'-bipyridine was prepared according to the reported procedure. ${ }^{10}$

\section{Measurements}

The ${ }^{1} \mathrm{H}$ and ${ }^{13} \mathrm{C}$ NMR spectra were recorded on a JEOL JNM-400II spectrometer in deutero-chloroform at $25^{\circ} \mathrm{C}$. The optical rotatory measurements were performed in chloroform at $28^{\circ} \mathrm{C}$ using a Jasco DIP 1000 digital polarimeter. The molecular weights were measured by gel permeation chromatography (GPC) in THF using a Jasco GPC 900 system equipped with three polystyrene columns (Shodex KF-804L). The number-average molecular weights $\left(M_{\mathrm{n}}\right)$ and polydispersity indices $\left(M_{\mathrm{w}} / M_{\mathrm{n}}\right)$ were calculated on the basis on a polystyrene calibration. The chiral high-performance liquid chromatography (HPLC) analysis was performed using a Jasco HPLC system (PU-980 Intelligent HPLC pump and UV 975 Intelligent UV detector) equipped with a Daicel CHIRALCEL OB-H column (hexane/2- 
propanol (vol. ratio 100/1) as the eluent, flow rate of $\left.0.5 \mathrm{~mL} \mathrm{~min}^{-1}\right)$.

\section{Rac-2,4-Pentanediyl Dimethacrylate}

The synthesis of $r a c-2,4$-pentanediyl dimethacrylate (rac-1) was reported in a previous paper.

\section{Polymerization of Rac-1 Using $\mathrm{AIBN} / \mathrm{Cu}(\mathrm{OTf})_{2} / 3$}

In a glove box (under a moisture- and oxygen-free argon atmosphere, $\left.\mathrm{H}_{2} \mathrm{O}, \mathrm{O}_{2}<1 \mathrm{ppm}\right)$, a mixture of rac-1 $(500 \mathrm{mg}, 2.1 \mathrm{mmol})$, AIBN $\left(1.7 \mathrm{mg}, 1.0 \times 10^{-2}\right.$ $\mathrm{mmol}), \mathrm{Cu}(\mathrm{OTf})_{2}\left(7.3 \mathrm{mg}, 2.1 \times 10^{-2} \mathrm{mmol}\right), 3(19 \mathrm{mg}$, $\left.4.2 \times 10^{-2} \mathrm{mmol}\right)$, and anisole $(21 \mathrm{~mL})$ was prepared and $2.0 \mathrm{~mL}$ of the mixture was transferred to a dry test tube. The capped test tubes were stirred at $60^{\circ} \mathrm{C}$ outside the glove box. At the end of the polymerization, a $20 \mu \mathrm{L}$ aliquot of the reaction mixture was added to hexane $(0.5 \mathrm{~mL})$ and filtered through a $0.25 \mu \mathrm{m}$ pore membrane filter. These samples were analyzed for monomer conversion and enantiomeric excess (e.e.) by HPLC equipped with a CHIRALCEL OB-H column, and for $M_{\mathrm{n}}$ and $M_{\mathrm{w}} / M_{\mathrm{n}}$ by GPC. The residual polymerization mixtures were passed through a short alumina column to remove the metal salts, and the solvent was removed under reduced pressure. The residue was poured into hexane and the precipitate was then filtered. The obtained powders were purified by reprecipitation using chloroform-methanol and dried in vacuo.

\section{REFERENCES}

1. Y. Okamoto, K. Urakawa, K. Ohta, and H. Yuki, Macromolecules, 11, 719 (1978).

2. Y. Okamoto, K. Ohta, and H. Yuki, Macromolecules, 11, 724 (1978).

3. Y. Okamoto, K. Urakawa, K. Ohta, and H. Yuki, J. Polym. Sci., Polym. Chem. Ed., 19, 1385 (1981).

4. M. Sepulchre and N. Spassky, Makromol. Chem., 182, 2225 (1981).

5. M. Sepulchre, Makromol. Chem., 188, 1583 (1987).

6. Y. Okamoto, M. Nishikawa, T. Nakano, E. Yashima, and K. Hatada, Macromolecules, 28, 5135 (1995).

7. T. Nakano, N. Kinjo, Y. Hidaka, and Y. Okamoto, Polym. J., 31, 464 (1999).

8. M. Tsuji, R. Sakai, T. Satoh, H. Kaga, and T. Kakuchi, Macromolecules, 35, 8255 (2002).

9. K. Matyjaszewski and B. E. Woodworth, Macromolecules, 31, 4718 (1998).

10. W.-S. Lee, H.-L. Kwong, H.-L. Chan, W.-W. Choi, and L.-Y. Ng, Tetrahedron: Asymmetry, 12, 1007 (2001). 